\section{MEDICINA INTERNA Y ENFERMEDADES RARAS. TRANSICIÓN NIÑO-ADULTO}

\author{
Francesc Cardellach \\ Hospital Clínic. Universidad de Barcelona \\ ORCID iD: https://orcid.org/0000-0003-3286-3436 \\ fcardell@clinic.cat \\ Antònia Ribes \\ Hospital Clínic. Universidad de Barcelona \\ ORCID iD: https://orcid.org/0000-0002-2249-246X \\ aribes@clinic.cat
}

\author{
INTERNAL MEDICINE AND \\ RARE DISEASES. \\ CHILD-ADULT TRANSITION
}

Cómo citar este artículo/Citation: Cardellach, F. y Ribes, A. (2018). Medicina interna y enfermedades raras. Transición niño-adulto. Arbor, 194 (789): a460. https://doi.org/10.3989/ arbor.2018.789n3002

Recibido: 30 abril 2015. Aceptado: 13 mayo 2016.

RESUMEN: La mayoría de las enfermedades raras (ER) son de base genética, cursan con síntomas muy diversos y a menudo ocasionan un grado variable de discapacidad, especialmente los errores congénitos del metabolismo (ECM). El proceso de transición clínica de un paciente con una ER que alcanza la edad adulta pretende garantizar una atención sanitaria ininterrumpida, coordinada y psicológicamente apropiada mediante unidades expertas (UE) multidisciplinares. El especialista en medicina interna tiene un papel central, junto a los de nutrición y dietética, laboratorio especializado en ECM y otros especialistas (neurología, genética clínica, farmacia, oftalmología, obstetricia, psiquiatría, enfermería, trabajo social, con el apoyo de pediatría experimentada en ECM). Las UE deberían organizar la asistencia, desarrollar guías clínicas de diagnóstico, seguimiento y tratamiento, adaptar los servicios de farmacia, crear un registro de pacientes, establecer relaciones con las asociaciones de pacientes, desarrollar un programa científico formativo, establecer colaboraciones internacionales, facilitar la colaboración con la industria farmacéutica y mantener una actividad investigadora propia.

PALABRAS CLAVE: enfermedades raras; enfermedades metabólicas hereditarias; errores congénitos del metabolismo; transición niño-adulto en errores congénitos del metabolismo.
Copyright: (c) 2018 CSIC. Este es un artículo de acceso abierto distribuido bajo los términos de la licencia de uso y distribución Creative Commons Reconocimiento 4.0 Internacional (CC BY 4.0).

ABSTRACT: Most rare diseases (RD) are genetically based, occur with very different symptoms and may cause a different degree of disability, especially inborn errors of metabolism (IEM). The clinical transition process of a patient with a RD reaching adulthood aims to ensure uninterrupted, coordinated and psychologically-appropriate health care through multidisciplinary expert units (EU). The specialist in Internal Medicine has a central role, along with Nutrition and Dietetics, a specialized ECM laboratory, and other specialists (Neurology, Clinical Genetics, Pharmacy, Ophthalmology, Obstetrics, Psychiatry, Nursing, Social Work, with the support of pediatricians experienced in ECM). EU should organize the overall care, develop clinical guidelines for diagnosis, followup and treatment, adapt Pharmacy services, create patient registers, establish relationships with patients' associations, develop a scientific training program, establish international partnerships, facilitate collaboration with the Pharmaceutical Industry and have its own research activity.

KEYWORDS: rare diseases; inherited metabolic diseases; inborn errors of metabolism; child-adult transition in inborn errors of metabolism. 


\section{INTRODUCCIÓN}

Las enfermedades raras (ER) son entidades muchas de ellas de base genética (hasta un $80 \%$ tienen una transmisión genética) que cursan con síntomas muy diversos y a menudo ocasionan un grado variable de discapacidad (Palau, 2010). Por otra parte, otras muchas ER se diagnostican en la edad adulta y existen numerosos profesionales que, desde sus respectivos ámbitos de interés, tienen un nivel de experiencia en dichos procesos que les permite constituirse en unidades o grupos de referencia para los pacientes que los padecen.

La existencia de estructuras asistenciales que garanticen una atención clínica adecuada de los pacientes afectos de ER constituye a menudo un reto sanitario de gran magnitud. Su desarrollo e implementación requiere no solamente un esfuerzo e implicación de los recursos humanos (de tipo médico, de enfermería, de trabajo social, de investigadores, de asociaciones de pacientes, etc.), sino también un compromiso de los responsables de la gestión sanitaria, estructuras docentes, industria farmacéutica, organismos de investigación y mecenazgo.

A continuación se presentan y se analizan aquellos aspectos que se han tener en cuenta en la asistencia a pacientes adultos con enfermedades raras, con especial atención a los errores congénitos del metabolismo (ECM) o enfermedades metabólicas hereditarias.

\section{ENFERMEDADES RARAS EN PACIENTES ADULTOS}

Es difícil establecer diferencias substanciales entre las ER de la edad infantil y aquellas que afectan a la edad adulta, puesto que fundamentalmente se trata de formas clínicas diferentes, aunque la alteración genética sea la misma. En general, será en los primeros períodos de la vida cuando se presenten y desarrollen con mayor probabilidad. Sin embargo, cuando muchos de estos pacientes alcanzan la edad adulta, los problemas que acarreaban debido a su enfermedad de base siguen activos. Así, si una ER diagnosticada en la edad infantil había conllevado, por su gravedad, un grado de discapacidad avanzado de tipo neurológico, las necesidades para su atención cuando se llega a la edad adulta suelen requerir mayores prestaciones, tanto para el propio paciente como para sus cuidadores. Incluso, en este último caso, la dedicación al paciente suele ser absoluta y comporta una carga adicional de responsabilidad difícil de llevar a cabo a medida que pasan los años, por motivos evidentes. Si el paciente era tributario de una dieta específica, vital para su normal desarrollo y tratamiento de su enfermedad, puede que en el momento de alcanzar la edad adulta y con capacidad de decisión se generen conflictos con los progenitores ante una resistencia fácil de entender; o en ocasiones, es el propio paciente quien tiene que adoptar una responsabilidad sobre sí mismo no acostumbrada hasta entonces, lo cual le puede exigir un ejercicio de autocontrol y rigor nada fácil de asumir.

En el caso de la mujer, al llegar a la edad adulta estas pacientes pueden tener hijos sanos si se adoptan las medidas necesarias. Así, por ejemplo, aquellas con tratamiento dietético pero que quizás podían haber relajado un poco la dieta, deberán reiniciar un tratamiento estricto en períodos previos al embarazo y durante toda la gestación. Esta medida va a condicionar el normal desarrollo del feto y constituirá la garantía de un recién nacido libre de problemas, principalmente de tipo neurológico. En otras ocasiones, el consejo genético constituirá la base para evitar una enfermedad en la descendencia; es relativamente comprensible que esta situación genere desasosiego, depresión, hastío e incluso dificultades en las relaciones de pareja. En estos casos, el apoyo del psicólogo puede ser de gran ayuda para superar momentos clave en la vida personal de (la mayoría de veces) una paciente con riesgo de que su descendencia pueda resultar afectada si no adopta las medidas adecuadas.

Otras veces, al llegar a la edad adulta, el paciente asume una responsabilidad sobre su proceso que le vuelve receloso hacia la atención por parte del profesional, y se vuelca en una búsqueda desesperada de información a través de los numerosos medios (principalmente Internet) hoy en día al alcance de cualquiera mínimamente experto en su manejo; tal interés no significa que sea intrínsecamente perverso pero, si no se sabe conducir con habilidad, la relación con el facultativo se desenvuelve en un clima de desconfianza que en nada contribuye al buen control del paciente. Esta circunstancia no es exclusiva del adulto, pues durante el período infantil puede suceder lo mismo, pero con los padres. En cualquier caso, hoy en día el profesional puede enfrentarse con este problema ante cualquier paciente adulto, tenga una ER u otro tipo de proceso, si bien el hecho de ser una ER (con el desconocimiento a menudo de su causa y de su tratamiento) probablemente se preste a mayor discusión.

Una problemática que a menudo se plantea con los sujetos adultos afectos de ER, especialmente si estos no son atendidos en centros de referencia, o bien en hospitales distintos a aquellos en los que venían siendo atendidos hasta entonces, es la accesibilidad a determinados tratamientos, ya sea por su complejidad o por su coste. Efectivamente, durante el período infantil es normal que el hospital de referencia haya constitui- 
do hasta entonces el marco asistencial habitual, pero al alcanzar la edad adulta se tiende a derivar a estos pacientes al médico de asistencia primaria, a menudo sin experiencia sobre dichas enfermedades. Es entonces cuando lo que parecía fácil se vuelve difícil debido a un entorno organizativo no apropiado, y las dificultades administrativas pueden, de forma involuntaria, pasar por delante de las necesidades del enfermo. Es por ello por lo que las estructuras sanitarias deben contemplar ( $y$, de hecho, en la mayoría de casos ya se contemplan) estos desajustes, y procurar que estos pacientes sean atendidos en hospitales preparados para ello, o bien mejorar la organización estructural del sistema sanitario para darles cobertura a través de una estrecha conexión entre sus distintos niveles (hospitales de tercer nivel, hospitales de segundo y primer nivel y centros de asistencia primaria). En ello juegan un papel relevante los propios profesionales, ya que deben tomar la iniciativa de establecer dicha relación, siempre con el apoyo de políticas sanitarias específicamente dirigidas en este sentido. Esta circunstancia de interrelación y colaboración se refiere no solo a los tratamientos (sean o no complejos), sino que es extensible incluso a las visitas ambulatorias que no tengan una dificultad específica que requiera la atención del especialista, quizás ubicado lejos del domicilio del sujeto afectado.

Finalmente, una de las cuestiones más importantes que se plantea en pacientes con ER en edad infantil que alcanzan la edad adulta es el hecho de que este momento es un período crucial en muchos sentidos. Aunque no necesariamente tiene que ser así, lo habitual es que estos enfermos sean atendidos a partir de este momento por otro profesional, no pediatra, por lo general desconocido para ellos y quizás en otro centro diferente al habitual hasta entonces. Este hecho, entre otros, que coincide con una etapa crucial del adolescente, ha sido tratado y analizado en numerosos foros y ha merecido la consideración de muchos expertos hasta establecer una serie de normas que la mayoría de las sociedades científicas y profesionales han hecho suyas (Blum et al., 1993; Buchbinder, Berger, Robert y Vannier, 2011).

\section{ATENCIÓN CLÍNICA EN EL PERÍODO DE TRANSICIÓN DE LA EDAD INFANTIL A LA ADULTA}

La transición clínica de un paciente con una ER que alcanza la edad adulta constituye un período que adquiere una gran relevancia por el contexto habitualmente de riesgo en que se produce (una edad sometida a la influencia de numerosos factores, psicológicos, educativos, sexuales, etc.) y por los cambios que necesa- riamente hay que adoptar para minimizar sus posibles consecuencias adversas. Ello requiere una gran atención por parte de los profesionales que están atendiendo al paciente y también por parte de aquellos que a partir de este momento van a tener cuidado del mismo, lo cual comporta la adopción de una serie de medidas que están bien establecidas bajo el término de transición.

La transición, en este contexto del ámbito médico, se define por el desplazamiento de adolescentes y jóvenes afectos de trastornos de tipo crónico desde un sistema centrado en el cuidado del paciente en edad infantil hasta otro orientado a la atención del sujeto adulto (Blum et al., 1993; Kossoff, Veggiotti, Genton y Desguerre, 2014). El objetivo final de dicha transición es que la atención sanitaria se lleve a cabo de una forma ininterrumpida, coordinada, adecuadamente desarrollada, psicológicamente apropiada y que sea debidamente comprendida. Si bien hasta hace unos años no se prestaba tanta atención a este proceso, el hecho de que en la actualidad prácticamente un $85 \%$ de pacientes en edad infantil alcancen la edad adulta ha condicionado que se deba recurrir a la adopción de unas medidas antaño impensables (por innecesarias). Ello adquiere mayor relevancia por cuanto la mayoría de los sistemas de salud están reforzando el papel de la medicina de familia, es decir, la atención que se recibe en aquellos centros de asistencia primaria más cercanos al lugar de vivienda habitual.

Otra cuestión que hay que tener en cuenta es que no siempre se produce una necesaria interrupción de la asistencia clínica por parte del profesional o profesionales que atendían al joven con la ER a partir del momento de su traspaso al equipo médico de adultos. Efectivamente, puede haber muy distintos modelos de transición, que van desde su derivación a otro centro (hospitalario o de atención primaria) hasta una continuidad garantizada por la presencia en el mismo centro de parte del equipo anterior juntamente con el nuevo. Por consiguiente, las dificultades en un caso y en otro pueden ser totalmente distintas en intensidad y calidad. Por ello, los aspectos que se comentan a continuación deben entenderse como generales y adaptables a cada circunstancia.

Para el proceso de transición es preciso tener en cuenta una serie de etapas bien establecidas:

\subsection{Información completa de la historia clínica del paciente al centro receptor}

Este es un aspecto en el que a todas luces parece innecesario insistir, aunque la experiencia ha demostrado que en la mayoría de las relaciones en el ámbito de 
la salud la falta de información (a menudo de forma involuntaria) es un factor clave para el buen desarrollo de una asistencia clínica de calidad. Es decir, antes de involucrar al paciente en cualquier proceso de derivación hay que asegurarse que la información acerca del mismo ya está en manos del equipo receptor. Una vez aceptada la recepción, es el momento de seguir adelante con el proceso.

\subsection{Proceso de transición}

El proceso de transición ha de ser:

a) Individualizado. Efectivamente, cada paciente es diferente y sus condiciones (físicas, psicológicas, del entorno, etc.) pueden aconsejar un tipo de actitudes y no otras.

b) Acordado. Dicho acuerdo debe ser total, y además con el compromiso tanto del propio paciente como de sus familiares o cuidadores.

c) Debe tener lugar fuera de períodos de crisis. El momento de la transición no se debe llevar a cabo en un período con intensos factores potencialmente desestabilizantes, con lo cual ante la presencia de cualquiera de ellos habría que retrasar el inicio de la transición.

d) Debe tener lugar al final del período docente. Es decir, cuando han finalizado las clases y no existe una situación de estrés que interfiera en el proceso de transición.

e) Debe tener en cuenta todas las necesidades sanitarias. Cada paciente puede requerir la atención inmediata de uno o más especialistas o de ninguno en particular, y en este caso el papel del médico internista (e incluso del médico de familia) adquiere una relevancia fundamental; más adelante se insistirá ampliamente en ello.

f) Debe tener en cuenta los aspectos económicos. Aunque puede resultar doloroso como aspecto que hay que considerar ante la salud de un paciente con una ER, este es un factor que siempre hay que dejar solucionado antes de proceder a su derivación. Si se lleva a cabo de forma adecuada, es posible evitar interrupciones del tratamiento que a veces resultan cruciales para su supervivencia. Una correcta coordinación de estos aspectos administrativo-económicos nunca hay que considerarla secundaria en el proceso de transición, sino que adquiere a menudo una relevancia que supera incluso cualquier otra consideración.

\subsection{Acciones que hay que llevar a cabo con el joven paciente antes de la derivación}

Para que la transferencia resulte más fácil al paciente, es necesario antes de la derivación:

a) Informarle del proceso. Es aconsejable que la información acerca de lo que significa este momento le llegue de forma progresiva y detallada, mucho antes de iniciar la ejecución de la transferencia (alrededor de los 12-14 años). Ello le permitirá hacerse una idea de todo el proceso asistencial con antelación, lo que le dará tiempo para plantearse todo tipo de preguntas y para obtener las respuestas más adecuadas.

b) Que el líder del grupo o su médico directamente responsable le comunique las etapas del proceso. Las etapas del proceso deben serle comunicadas por un profesional con experiencia, a ser posible por el facultativo que ha venido teniendo la responsabilidad de su control durante años.

c) Asignarle un profesional de trabajo social. Esta persona debería mantener una estrecha relación con el paciente de forma continuada e incluso estar presente en las visitas del último año antes de su trasferencia (o el tiempo que se considere necesario).

d) Proporcionarle un plan global de transferencia. El enfermo debería disponer de una agenda con control de los tiempos y consecución de determinadas competencias o habilidades con relación al proceso que garanticen una preparación suficiente para llevar a cabo la transferencia sin dificultades.

e) Incluir acompañantes. Estos pueden ser los padres, otros familiares, amigos o pareja del joven; cuanta más información y menos incertidumbre entre el propio enfermo y también entre sus acompañantes, más garantía de que el proceso se llevará a cabo con éxito.

f) Visitar el centro receptor. Siempre es conveniente conocer con antelación el centro o equipo receptor, puesto que sin la angustia de la primera visita médica este acercamiento es mucho mejor aceptado y crea menos ansiedad.

g) Facilitarle la reunión con otros jóvenes inmersos en el proceso de transición. La participación del joven en reuniones, conversaciones, contactos (telefónico, presencial, por correo electrónico) con otros jóvenes prácticamente ya en proceso de transferencia le permitirá detectar con mayor facilidad aquellos aspectos más comprometidos y hacerles frente con mayores garantías de éxito. 
h) Facilitarle guías de su enfermedad. Proporcionar información escrita sobre su proceso le ayudará a adquirir confianza en sí mismo y en las acciones que el nuevo equipo o profesional deberá adoptar en esta nueva fase.

\subsection{Características de los grupos o unidades de atención a pacientes adultos con ER}

Este apartado concierne a una cuestión en la que, de modo genérico, existe amplio consenso en cuanto a determinados aspectos generales que deben cumplir la mayoría de los grupos o unidades de atención a pacientes adultos con ER. Sin embargo, dependiendo del tipo de ER es lógico suponer que algunas cuestiones específicas deberán ajustarse a cada una de las mismas. Por consiguiente, dada la variedad y número de aquellas, el detalle propio de forma individualizada queda fuera de los objetivos de estas páginas. A modo de ejemplo, y también por su elevada prevalencia, a continuación se exponen las características más relevantes de una unidad de atención a pacientes adultos con errores congénitos del metabolismo (ECM); estas enfermedades constituyen un paradigma de proceso crónico, la mayoría se diagnostican al nacer o al poco tiempo de vida, precisan de atención multidisciplinar y representan un ejemplo para ilustrar el proceso de transición comentado más arriba (Lee, 2002; Mütze et al., 2011). Por otro lado, es de destacar el papel aglutinador que el especialista en medicina interna puede llevar a cabo en el seno del equipo.

\section{COMPOSICIÓN DE LOS GRUPOS O UNIDADES DE PACIENTES ADULTOS CON ERRORES CONGÉNITOS DEL METABOLISMO (ECM). PAPEL DE LA MEDICINA INTERNA COMO FACTOR AGLUTINADOR DE LA LA- BOR ASISTENCIAL}

Los ECM son un grupo muy numeroso de enfermedades (véase la tabla 1), dentro de las ER. Se caracterizan por ser detectables a nivel bioquímico (por análisis de metabolitos o por estudios enzimáticos o, en general, por estudios de la proteína alterada). Las manifestaciones clínicas en la mayoría se inician ya en el período neonatal o durante la lactancia, mientras que otras lo hacen más adelante, e incluso en la edad adulta. Sin embargo, los avances en el diagnóstico y tratamiento de muchos de estos procesos, así como la implantación del cribado neonatal, han hecho posible que cada vez con mayor frecuencia se pueda establecer un diagnóstico inmediato y poner en marcha las medidas necesarias para evitar consecuencias que de otro modo pueden llegar a ser irreversibles o incluso producir la muerte. Por consiguiente, cada vez es más habitual que muchos pacientes alcancen la edad adulta, momento en el cual se plantean diversas acciones que conviene llevar a cabo para ofrecer la misma calidad asistencial que hasta entonces venían recibiendo por parte de los profesionales de los servicios de Pediatría. Esta situación genera unas necesidades de diversa índole que deben tener en cuenta el momento crucial, en muchos aspectos, que representa el cambio asistencial planteado por el sistema organizativo de la estructura sanitaria.

El diagnóstico precoz de los ECM permite, en muchas ocasiones, establecer un tratamiento adecuado y con ello se pueden evitar complicaciones graves con secuelas irreversibles, la mayoría de las cuales y las de peor pronóstico son de tipo neurológico. En el momento actual el tratamiento de muchos de estos pacientes se fundamenta en la observación rigurosa de una dieta específica (distinta según el tipo de proceso), en la toma de determinados fármacos que convierten en funcional la proteína alterada o bien en la administración de la enzima deficitaria (terapia enzimática sustitutiva). Otros tratamientos más específicos están en fase de investigación (terapia génica y celular, trasplante hepático). Sin embargo, en otras ocasiones el tratamiento se limita al alivio de los síntomas.

En este contexto, la composición de las unidades o grupos al cuidado de estos pacientes tiene que emular en gran medida a la que se venía observando en la edad infantil, ciertamente teniendo en cuenta determinadas consideraciones (Lee, 2002; Mütze et al., 2011). En primer lugar, una de las características más llamativas de los ECM es la afectación de diversos aparatos y sistemas, aunque a menudo la más temible es la de tipo neurológico por las consecuencias potencialmente graves sobre el sistema nervioso central. Otra consideración es que los pacientes ya han superado la fase de crecimiento y desarrollo físico, con lo cual es probable que el control de determinados parámetros, y por tanto el papel de algunas especialidades, se vea aliviado en favor de una mayor intervención por parte del médico internista. En efecto, la mayoría de pacientes adultos con ECM han sido diagnosticados en la edad infantil, por lo que sus procesos, en general, suelen estar bien establecidos y su tratamiento correctamente determinado, lo que les permite alcanzar aquella edad en una situación de estabilidad. Es precisamente por ello por lo que el internista con una formación adecuada debe disponer de la capacidad de mantener esta estabilidad, y al mismo tiempo detectar cualquier cambio (clínico o analítico) que sugiera la aparición más o menos próxima de alguna descompensación de su enfermedad. En este caso, el papel del especialista se hace absolutamente necesario. 
Tabla 1. Clasificación de los Errores Congénitos del Metabolismo (ECM)

\section{ENFERMEDADES LISOSOMALES}

Esfingolipidosis

Gangliosidosis GM1

Gangliosidosis GM2

Enfermedad de Tay-Sachs y variante B1

Enfermedad de Sandhoff

Gangliosidosis GM2 por déficit de proteína activadora

Enfermedad de Gaucher

Enfermedad de Gaucher por déficit del activador SAP-2

Enfermedad de Niemann-Pick tipo A

Enfermedad de Niemann-Pick tipo B

Enfermedad de Niemann-Pick tipo C

Enfermedad de Fabry

Leucodistrofia metacromática

Leucodistrofia metacromática por déficit de SAP-1

Deficiencia múltiple de sulfatasas

Leucodistrofia de Krabbe

Mucopolisacaridosis

Síndromes de Hurler, Hurler-Scheie y Scheie (MPS I)

Síndrome de Hunter (MPS II)

Síndromes de Sanfilippo, A, B, C y D (MPS III A, B, C y D)

Síndromes de Morquio A y $B$ (MPS IV A y B)

Síndrome de Maroteaux-Lamy (MPS VI)

Deficiencia de ß-glucuronidasa o Síndrome de Sly (MPS VII)

Glucoproteinosis

$\alpha$-Manosidosis

ß-Manosidosis

$\alpha$-Fucosidosis

Sialidosis

Galactosialidosis

Aspartilglucosaminuria

Enfermedad de Schindler

Defectos en el procesamiento y transporte de las Hidrolasas ácidas

Enfermedad de las células con inclusiones (Mucolipidosis II)

Polidistrofia pseudo-Hurler (Mucolipidosis III)

Defectos en la salida de metabolitos del lisosoma por alteración del transporte proteínas

Enfermedad de Salla

Enfermedad por almacenamiento de ácido siálico, forma infantil

Otras enfermedades lisosomales

Deficiencia de $\alpha$-glucosidasa o Enfermedad de Pompe Enfermedad de Wolman

Enfermedad por almacenamiento de ésteres del colesterol Ceroide lipofuscinosis neuronal, infantil (CLN1)

Ceroide lipofuscinosis neuronal, infantil tarde (CLN2)

Picnodisostosis

\section{ENFERMEDADES PEROXISOMALES}

Trastornos de la biogénesis peroxisomal

Síndrome de Zellweger (SZ)

Adrenoleucodistrofia neonatal (ALDN)

Enfermedad de Refsum infantil (IR)

Condrodisplásia punctata rizomélica autosómica recesiva (CDPR)
Defectos aislados de las vías peroxisomales

Adrenoleucodistrofia ligada al X (X-ALD/AMN)

Seudo-adrenoleucodistrofia neonatal (PALDN)

Deficiencia de proteína bifuncional

Seudo-Zellweger (PSZ)

Deficiencia de DHAPAT

Deficiencia de alquil DHAP sintasa

Enfermedad clásica de Refsum

\section{TRASTORNOS DEL METABOLISMO DE LOS ESTEROLES}

Síndrome de Smith-Lemli-Opitz (SLO)

Xantomatosis Cerebrotendinosa (XCT)

Desmosterolosis (DS)
Condrodisplásia punctata rizomélica ligada a $\mathrm{X}$ dominante (CDPRX2)

Síndrome CHILD

Sitosterolemia (ST) 


\begin{tabular}{|c|c|}
\hline \multicolumn{2}{|l|}{ ACIDURIAS ORGÁNICAS Y AMINOACIDOPATÍAS } \\
\hline $\begin{array}{l}\text { Acidemia propiónica } \\
\text { Enfermedad de "Jarabe de Arce" } \\
\text { Acidemias metilmalónicas } \\
\text { Acidemia isovalérica } \\
\text { Deficiencia de 3-metilcrotonil-CoA carboxilasa } \\
\text { Acidurias 3-metilglutacónicas } \\
\text { Aciduria 3-hidroxi-3-metil glutárica } \\
\text { Deficiencia de 2-metil-acetoacetil-CoA tiolasa } \\
\text { Aciduria 3-hidroxi-isobutírica } \\
\text { Deficiencia de biotinidasa } \\
\text { Deficiencia de holocarboxilasa sintetasa } \\
\text { Aciduria glutárica tipo I } \\
\text { Aciduria 2-hidroxiglutárica } \\
\text { Aciduria 2-metil-3-hidroxibutírica } \\
\text { Aciduria 4-hidroxibutírica } \\
\text { Aciduria mevalónica } \\
\text { Aciduria N-acetilaspartica (Enfermedad de Canavan) } \\
\text { Deficiencia de glutation sintetasa } \\
\text { Deficiencia de 5-oxoprolinasa } \\
\text { Hiperoxaluria tipo I } \\
\text { Hiperoxaluria tipo II } \\
\text { Aciduria D y L glicérica } \\
\text { Alcaptonuria }\end{array}$ & $\begin{array}{l}\text { Tirosinemia tipo I } \\
\text { Tirosinemia tipo II } \\
\text { Hiperglucémia no cetósica } \\
\text { Hiperornitinémia } \\
\text { Hiperfenilalaninemias y Fenilcetonúria } \\
\text { Histidinemia } \\
\text { Trastornos del metabolismo de la Prolina e Hidroxiprolina } \\
\text { Hiperlisinemias } \\
\text { Trastornos de la degradación de la ß-alanina y del ácido } \\
\text { 4-amniobutírico } \\
\text { Homocistinurias } \\
\text { Cistationinuria } \\
\text { Deficiencia del Cofactor Molibdeno } \\
\text { Cistinurias } \\
\text { Iminoglicinuria familiar } \\
\text { HiperaminoAciduria dibásica } \\
\text { Enfermedad de Hartnup } \\
\text { Enfermedad de Lowe } \\
\text { Cistinosis } \\
\text { Hiperornitinemia con Hiperamoniemia y Homocitrulinúria } \\
\text { (HHH) } \\
\text { Intolerancia a las proteínas con lisinuria (LPI) }\end{array}$ \\
\hline DEFICIENCIAS DEL CICLO DE LA UREA & DEFICIENCIAS DE CREATINA CEREBRAL \\
\hline $\begin{array}{l}\text { Deficiencia de carbamil fosfato sintetasa (CPS) } \\
\text { Deficiencia de ornitina carbamil transferasa (OCT) } \\
\text { Citrulinemia } \\
\text { Aciduria argininsuccínica } \\
\text { Argininemia }\end{array}$ & $\begin{array}{l}\text { Deficiencia de arginina: glicina amidinotransferasa (AGAT) } \\
\text { Deficiencia de guanidinoacetato metiltransferasa (GAMT) } \\
\text { Deficiencia del transportador de creatina (CrT) }\end{array}$ \\
\hline DEFICIENCIAS DE LA ß-OXIDACION MITOCONDRIAL & $\begin{array}{l}\text { DEFICIENCIAS DEL METABOLISMO DEL PIRUVATO Y DE LA } \\
\text { CADENA RESPIRATORIA MITOCONDRIAL }\end{array}$ \\
\hline $\begin{array}{l}\text { Deficiencia primaria de carnitina (CUD) } \\
\text { Deficiencia de carnitina palmitoiltransferasa I (CPT-I) } \\
\text { Deficiencia de carnitina palmitoiltransferasa II (CPT-II) } \\
\text { Deficiencia de acilcarnitina translocasa (CACT) } \\
\text { Deficiencia de acil-CoA deshidrogenasa de cadena corta (SCAD) } \\
\text { Deficiencia de acil-CoA deshidrogenasa de cadena media } \\
\text { (MCAD) } \\
\text { Deficiencia de acil-CoA deshidrogenasa de cadena muy larga } \\
\text { (VLCAD) } \\
\text { Deficiencia de 3-hidroxiacil-CoA deshidrogenasa de cadena } \\
\text { corta (SCHAD) } \\
\text { Deficiencia de 3-hidroxiacil-CoA deshidrogenasa de cadena } \\
\text { larga (LCHAD) } \\
\text { Deficiencia de proteína trifuncional mitocondrial (MTP) } \\
\text { Deficiencia múltiple de deshidrogenasas (MAD) } \\
\text { Deficiencia en la síntesis y transporte de flavina adenina } \\
\text { dinucleótido (FAD) }\end{array}$ & $\begin{array}{l}\text { Deficiencia de piruvato deshidrogenasa (PDH) } \\
\text { Deficiencia de fumarasa } \\
\text { Deficiencia de 2-cetoglutarato deshidrogensa } \\
\text { Deficiencia de Complejo I } \\
\text { Deficiencia de Complejo II } \\
\text { Deficiencia de Complejo II } \\
\text { Deficiencia de Complejo V } \\
\text { Deficiencias combinadas de los complejos de la cadena } \\
\text { respiratoria } \\
\text { Depleciones de mtDNA } \\
\text { Deficiencias primarias de CoQ10 } \\
\text { Otras alteraciones de la cadena respiratoria mitocondrial }\end{array}$ \\
\hline
\end{tabular}


Deficiencia de galactosa-1-fosfato uridil transferasa

(Galactosemia clásica)

Deficiencia de galactoquinasa

Deficiencia de UDP galactosa-4-epimerasa
Defectos congénitos de la N-glicosilación

Defectos congénitos de la O-glicosilación

Defectos congénitos de la $\mathrm{N}$ y O-glicosilación

OTRAS ENFERMEDADES

Ictiosis ligada al $\mathrm{X}$

Con todo, la composición de la unidad debe ser multidisciplinar (Izquierdo Martínez y Avellaneda Fernández, 2003), puesto que es la mejor forma de compartir opiniones y tomar decisiones acertadas en favor del paciente con un ECM.

\subsection{Especialista en Medicina interna}

En nuestra opinión, y también según la experiencia de otros grupos, la referencia al especialista en Medicina Interna como protagonista obligado en la atención a pacientes con ER, y en particular a los pacientes con ECM, es totalmente oportuna. Por consiguiente, el papel central del internista, debidamente formado, está claramente justificado y con toda seguridad debería abarcar el mayor tiempo de la asistencia clínica de los pacientes con ECM por parte del equipo multidisciplinar (Orden SCO/227/2007 de 24 de enero).

Para mantener la continuidad asistencial en la atención de todos estos pacientes, el internista ha de estar especialmente preparado para trabajar en equipo en colaboración con otros especialistas hospitalarios, con el médico de familia, y con otros profesionales sanitarios (Cardellach y Vilardell, 2006). Tampoco puede olvidarse que, cada vez más, muchos de estos pacientes diagnosticados en el período infantil alcanzan la edad adulta con dificultades para identificar a su médico de referencia. Con todo ello se pretende indicar que el futuro especialista en Medicina Interna se debería convertir en el interlocutor entre los médicos de familia y los distintos especialistas hospitalarios, expertos en alguna de estas ER, absolutamente necesarios para atender algunas de las complicaciones que estos pacientes puedan presentar durante su evolución. Además, las labores asistenciales del internista se deberían complementar con las investigadoras, en el más puro sentido de investigación traslacional.

Una tercera consideración en cuanto al papel aglutinador del equipo por parte del internista en la atención a pacientes con ECM (y otras ER), es precisamente el reto de conseguir una coordinación entre sus distintos miembros (Grau y Cardellach, 2010; Socie- dad Española de Medicina Interna). Los enfermos, durante toda su etapa infantil, han gozado de una atención clínica de gran calidad y al mismo tiempo muy bien sincronizada entre los diversos especialistas, que facilitó enormemente el proceso en cada visita. Es, por consiguiente, necesario que no se pierda este grado de excelencia pero, al mismo tiempo, que el joven se habitúe a asumir una responsabilidad progresiva como adulto y entienda que a partir de este momento, y cada vez más, las decisiones se van a adoptar de común acuerdo con su opinión, cada vez más independiente de la de sus padres o mentores. En esta decisión, obviamente, es fundamental el grado de madurez e indemnidad neurológica del afectado.

\subsection{Facultativos de laboratorio}

Los facultativos de laboratorio han de tener formación específica en ECM. Su relevancia y papel fundamental en el diagnóstico y control del tratamiento de los enfermos con ECM es de suma importancia. La labor asistencial, docente e investigadora de un laboratorio de estas características es imprescindible para el normal funcionamiento de una unidad que atienda a estos pacientes. La interacción o feed-back entre profesionales clínicos (al lado del paciente) y el laboratorio aumenta la calidad de la asistencia, al hallarse todos ellos más sensibilizados con el problema, y además el análisis de cualquier anomalía o accidente clínico resulta mucho más rentable a la hora de encontrar la solución más adecuada.

\subsection{Especialistas en nutrición y dietética}

Su papel es relevante y absolutamente necesario, puesto que ejercen el control nutricional y dietético de ciertos ECM.

\subsection{Otros profesionales especialmente sensibilizados en ER}

También es necesario el concurso de otros especialistas que puedan dar respuesta a cualquier eventualidad que surja al margen de las habituales 
y que por su complejidad aconseje su concurso. Así, es muy deseable la participación de profesionales de Genética Clínica, Farmacia, Oftalmología, Obstetricia, Psiquiatría y Neurología. Estos especialistas deben estar dispuestos a responder de forma ágil y responsable a cualquier consulta que se les reclame dentro de su ámbito.

\subsection{Profesionales de Enfermería}

El papel de la enfermería en estas unidades de adultos con ECM, al igual que en estructuras similares para otras ER, es fundamental por cuanto su interacción inmediata y experiencia acerca de las necesidades de estos pacientes sobrepasa la atención puramente médica. El rol de enfermería, no solo en la atención asistencial inmediata, sino también a través de correo electrónico o contacto telefónico, en situaciones de consultas imprevistas, es muy relevante, ya que su experiencia permite solventar numerosas cuestiones que probablemente no precisen de una visita médica concertada. Su capacidad profesional en el respectivo ámbito (conocimiento de las necesidades de medicación, relaciones conflictivas, curas, fisioterapia, rehabilitación, extracciones de muestras de sangre, orina y saliva, etc.) les habilita para una actividad específica y muy efectiva. Una de las actividades más relevantes en las unidades de adultos con ER es el denominado gestor de caso, un profesional especializado (habitualmente del ámbito de enfermería) en las cuestiones más habituales que atañen a una determinada ER. Del mismo modo, cabe comentar también el papel de los profesionales de trabajo social, por cuanto muchas de estas enfermedades ocasionan desequilibrios familiares o bien el propio paciente con riesgo de exclusión del entorno precisa de algún tipo de ayuda social o de otro tipo (económico, administrativo, consejo, etc.).

\subsection{Pediatra experimentado en ECM}

La inclusión de un pediatra es a todas luces aconsejable, por un lado, para que el joven no se sienta bruscamente desatendido por su médico habitual de referencia, y por otro porque la experiencia de este profesional en el ámbito de los ECM es habitualmente muy consolidada y por consiguiente su aportación en todo momento es de gran interés asistencial. Sin embargo, no siempre es posible esta conjunción de profesionales y ello dependerá de las características del centro, de la labor específica de aquellos dentro del mismo y de la capacidad de motivación y convencimiento por parte del coordinador de la unidad.

\subsection{Médicos residentes}

Para garantizar la continuidad asistencial de una unidad de atención a pacientes adultos con ER (en este caso, ECM), es preciso elaborar un programa docenteformativo que incluya la participación (rotación) de médicos residentes y que necesariamente incluyan el laboratorio y el mismo servicio de Pediatría. La multiplicidad de órganos y sistemas afectados en las enfermedades metabólicas congénitas constituye un marco apropiado para la formación del residente en medicina interna y seguramente también para otras especialidades. Solamente de esta manera se pueden fidelizar profesionales con vocación de atender a pacientes con ECM u otras ER. El papel de los servicios de medicina interna en la consecución de este objetivo, y el de las unidades de pacientes con ECM, es crucial para ofrecer una atención asistencial adecuada.

\section{OBJETIVOS DE LOS GRUPOS O UNIDADES DE PA- CIENTES ADULTOS CON ERRORES CONGÉNITOS DEL METABOLISMO (ECM)}

Independientemente de que el motivo último de la creación de unidades de atención a pacientes adultos con ECM sea fundamentalmente proveer una asistencia clínica de la mejor calidad, no se puede olvidar que esta solamente se conseguirá a través de la consecución de una serie de objetivos que se detallan a continuación.

Objetivo 1. Reconocimiento oficial por parte de las estructuras directivas y organizativas del centro y servicios de salud. Una unidad de estas características debe poseer el apoyo oficial para poder desarrollarse y disponer de las mismas oportunidades que otras estructuras asistenciales; en el caso contrario, la situación de la misma estaría sustentada en falso y no podría ofrecer una asistencia adecuada. Por consiguiente, es aconsejable que su creación vaya precedida de, en primer lugar, una necesidad evidente, y posteriormente, de todos los pasos administrativos necesarios para conseguir el reconocimiento dentro del organigrama asistencial.

Otro nivel muy importante de reconocimiento de estas unidades debe ser lograr la consideración de unidades expertas (CatSalut, Instrucció 12/2014). El logro de esta consideración solamente puede provenir del correcto funcionamiento desde el punto de vista asistencial con el apoyo de la investigación y la formación, tal como se comenta más adelante.

Con todo, ninguna estructura de este tipo se puede crear de la nada, es decir, sin la implicación y esfuerzo 
convencido de los propios profesionales implicados. Por lo tanto, solo con el firme convencimiento de los mismos, muy probablemente asociado a una mayor dedicación y esfuerzo personal sin esperanza de retorno concreto a cambio, salvo el sentimiento de profunda satisfacción de raíz vocacional, se podrá conseguir crear una unidad de atención a pacientes adultos con $E R$, en este caso ECM.

Objetivo 2. Desarrollo o adaptación de guías clínicas de diagnóstico, de seguimiento, y sobre todo de tratamiento de los ECM en pacientes adultos. Efectivamente, en general existe amplio consenso acerca de la actitud que se debe seguir en estos aspectos en el caso de la edad infantil, pero ello está menos establecido en el caso de los adultos. Probablemente este hecho se deba a que muchos de estos enfermos hace unos años no alcanzaban la edad adulta, pero los avances en el diagnóstico y tratamiento de estas enfermedades han invertido esta tendencia y cada vez son más los niños afectos que llegan a esta edad. Por ello, es fundamental adaptar las guías a sus necesidades. Y esta actitud debe contemplar también los tratamientos de situaciones urgentes, muy comunes en algunos tipos de ECM cuando sufren alguna descompensación. Todas estas guías y protocolos, una vez validados por los profesionales acreditados, deben estar en un lugar fácilmente accesible a disposición de todos los facultativos las veinticuatro horas del día. Los sistemas informáticos hoy en día permiten conseguir esta disponibilidad sin mayor problema.

Objetivo 3. Adaptación de los servicios de farmacia a estas nuevas necesidades, buscando el mejor equilibrio (si es preciso, en colaboración con otros centros cercanos) por lo que respecta al coste/eficiencia de los medicamentos más caros que se deben disponer en reserva.

Objetivo 4. Organización de la asistencia clínica. El seguimiento en régimen ambulatorio de los pacientes con ECM debería organizarse en consultas de tipo monográfico, es decir, sin la presencia de enfermos afectos de otros procesos (no ER). Ello favorecería la coordinación con otras especialidades y con el pediatra experto, así como la intervención de enfermería y trabajo social, lo que resultaría en una atención más focalizada hacia aquellos. Este objetivo, muy ambicioso en sí mismo, puede verse limitado en su logro debido a la existencia de inercias establecidas a lo largo de años, pero en todo caso jamás hay que renunciar a su consecución.

En este mismo ámbito es necesario disponer de facilidades para la obtención, traslado y procesamiento de muestras biológicas para la investigación y en este aspecto el papel de enfermería adquiere una relevancia especial. No debe olvidarse la necesidad de disponer de un documento de consentimiento informado (reconocido por el Comité Ético de Investigación Clínica) para facilitar los trámites al respecto; en cualquier caso, la frialdad de dicho documento no puede sustituir todo tipo de explicaciones al paciente o a sus acompañantes.

Otro aspecto importante en este apartado es la necesidad de establecer una relación estrecha con el centro hospitalario cercano al domicilio del paciente y con el médico de familia del centro de asistencia primaria correspondiente. Ello significa favorecer el intercambio de información personal entre el facultativo del centro de referencia y el médico de familia (nombre, teléfono, correo electrónico) y también compartir información médica y de cualquier otro tipo que ataña al paciente. El objetivo es que en cualquier momento este pueda acudir a uno u otro centro y que los distintos facultativos implicados sean conocedores de su proceso con el fin de adoptar las decisiones que se estimen oportunas en cada momento. Este intercambio de información redunda en beneficio de los pacientes, por cuanto mejora su confianza en los profesionales y en muchas ocasiones evita desplazamientos innecesarios.

Objetivo 5. Elaboración de perfiles de laboratorio para cada enfermedad metabólica. La gran diversidad de los ECM añade, al propio proceso, un factor de complejidad nada despreciable y por ello es aconsejable establecer unos perfiles de laboratorio adecuados a cada enfermedad, con el fin de facilitar la labor del profesional y obviar extracciones innecesarias o por el contrario un descuido evitable. Ello solo se puede llevar a cabo después de un análisis riguroso de cada proceso, de una revisión exhaustiva de los protocolos de seguimiento específicos de la edad pediátrica y del análisis de su adecuación al paciente adulto; solo así será posible establecer los parámetros de control imprescindibles y la periodicidad de los mismos. En esta decisión deben participar expertos en dichas enfermedades (incluso del ámbito pediátrico), responsables de laboratorio, gestores y también especialistas en informática médica. Este proceder garantizará una utilización razonable de los recursos.

Objetivo 6. Solicitud de otras exploraciones, la mayoría probablemente basadas en la imagen (radiología simple, densitometrías, resonancia magnética, etc.). Esta solicitud debería basarse en los criterios de necesidad y oportunidad, según aconsejen las guías en cada caso. 
Objetivo 7. Creación de un registro o base de datos. Toda unidad o grupo de trabajo en ECM del adulto debe disponer de un registro propio que contenga la mayor información posible de cada paciente, no solo desde el punto de vista médico, sino también administrativo. La inclusión de determinados datos administrativos permite conocer, por ejemplo, el origen del paciente o su lugar de vivienda habitual. Ello va a permitir establecer, en cualquier momento, la prevalencia de un determinado proceso en el territorio, así como los centros de asistencia más cercanos al lugar de residencia. En el primer caso, se contribuirá al conocimiento de la enfermedad, y en el segundo se dispondrá de un dato imprescindible para mejorar la coordinación entre los distintos niveles asistenciales (una prioridad repetidamente puesta de manifiesto por los responsables de la gestión de los sistemas de salud). Ambos son objetivos comunes que hay que conseguir en cualquier tipo de ER. La elaboración de estas bases de datos constituye el embrión de los registros de ECM (y de las ER en general), tanto en el nivel local como nacional e internacional.

Objetivo 8. Establecimiento de relaciones con las asociaciones de pacientes (y sus familiares). En el ámbito de las ER, y más específicamente en los ECM, el papel de estas asociaciones es muy relevante por motivos obvios. En efecto, en primer lugar, por la misma naturaleza del proceso, ya que a la propia esencia de su baja frecuencia se añade el hecho de que la enfermedad se presenta mayoritariamente en la edad infantil; ambas circunstancias son factores que estimulan la creación de este tipo de asociacionismo. Por otro lado, las asociaciones de pacientes constituyen un revulsivo necesario para llamar la atención a los gestores de los servicios de salud sobre la escasez de recursos que a menudo se invierten en la investigación, formación y atención sanitaria en ER. Además, la relación de estas asociaciones con los profesionales que atienden a estos pacientes fortalece la confianza entre ambos y abre caminos que a veces no podrían labrarse por otros medios. Finalmente, la presión de estas asociaciones de pacientes y familiares en distintos ámbitos es un factor determinante a la hora de incluir un determinado tratamiento (habitualmente muy caros por tratarse de medicamentos huérfanos) en la cartera de prestaciones de los servicios de salud.

Objetivo 9. Desarrollo de un programa científico y formativo. Una unidad de atención a pacientes adultos con ECM debe tener entre sus objetivos principales conseguir un elevado nivel científico y educativo. Sin ello no se puede garantizar una buena asistencia, y en consecuen- cia tal unidad no tendría ningún futuro. Por tal razón desde el principio deben tenerse muy presentes los tres ámbitos de actuación: asistencia, investigación y docencia.

La investigación clínica, con la colaboración de laboratorios especializados en ECM, es muy importante por cuanto es la más próxima a la asistencia y sus resultados comportan beneficios inmediatos a los pacientes. Sin embargo, es también muy útil y necesario, o cuando menos aconsejable, disponer de la capacidad de llevar a cabo aspectos de la investigación de tipo más básico. En este sentido, la colaboración activa de investigadores de otras ramas de la ciencia, como no puede ser de otro modo, complementa y garantiza un trabajo en equipo multidisciplinar.

El ámbito formativo es crucial para asegurar la calidad asistencial y la continuidad en el tiempo de una unidad de adultos en ECM. Así, un programa atractivo de rotación de médicos residentes (MIR) por la unidad y sus diversos dispositivos, que incluya la posibilidad de realizar la tesis doctoral al final del período formativo, debe constituir un aliciente para mantener actualizado el equipo de trabajo estable de la unidad y conseguir, al mismo tiempo, fidelizar jóvenes profesionales sensibilizados con las ER. Dicho programa formativo debe estar abierto a la participación de MIR de las distintas especialidades implicadas, tanto del propio centro como de otros.

Objetivo 10. Colaboración con otras unidades de adultos a nivel nacional e internacional. Esta colaboración es necesaria para la consolidación de cualquier unidad de este tipo, por lo que la información de la existencia de aquellas y el establecimiento de acciones conjuntas debe constituir un objetivo prioritario. La inscripción de los miembros en las sociedades científicas de este ámbito y la asistencia a reuniones y congresos facilita enormemente dicho objetivo.

Objetivo 11. Facilitación de las relaciones con la Industria farmacéutica. Estas relaciones son muy importantes tanto desde el punto de vista asistencial como de investigación. Efectivamente, uno de los problemas más habituales en pacientes con ECM es el tratamiento de determinados procesos que precisan medicaciones de elevado coste, pero que son la única posibilidad terapéutica para estos pacientes. Independientemente de las acciones institucionales con los gestores de los servicios de salud, en las que las unidades reconocidas pueden tener un papel de asesoramiento muy relevante en la síntesis, desarrollo y comercialización de fármacos, la inclusión de pacientes en ensayos clínicos puede facilitar la consecución de determinados fármacos. 
Por otro lado, la participación de la industria farmacéutica en proyectos de investigación, programas formativos, becas para jóvenes profesionales, etc. constituye otro ámbito de colaboración de aquella con las unidades de adultos con ECM.

Objetivo 12. Colaboración con las iniciativas de registros sobre ECM. La elaboración de un registro sobre una ER tiene una dificultad añadida a la propia de cualquier registro de otra enfermedad, y es precisamente la escasa prevalencia de aquella. Existen iniciativas de esta índole a diversos niveles, como autonómico, nacional, europeo e incluso mundial. Ya se ha tratado anteriormente la importancia de la confección de bases de datos para luego poder contribuir a la elaboración de los registros. Cualquier unidad de adultos en ECM debe ser consciente de que su papel va más allá de lo que significa un buen funcionamiento de aquella. Sin una aportación de la información que poseen estas unidades a los organismos que tienen como fin elaborar estos registros la consideración de unidad queda desvirtuada al evitar o dificultar el mejor conocimiento de una enfermedad determinada.

Objetivo 13. Desarrollo de actividad investigadora propia. Ya se ha tratado en otros apartados la importancia de llevar a cabo investigación en ECM para poder desarrollar una asistencia de calidad. Una unidad de adultos en este tipo de procesos probablemente se halle, al menos inicialmente, un paso por detrás de similares unidades en el ámbito pediátrico, puesto que la historia y, por consiguiente, la experiencia en este tipo de ER así lo justifican. Sin embargo, ello no puede ser una limitación para reducir el nivel de investigación en estas enfermedades cuando dichos pacientes alcanzan la edad adulta. El estudio de la etiología, fisiopatología, epidemiología, tratamiento, etc. está al alcance de todos los centros mínimamente preparados para atender a estos enfermos.

La aportación de muestras biológicas a los biobancos de los organismos e instituciones del ámbito universitario e investigador debe ser un objetivo prioritario. Para ello se deben seguir los protocolos de información, consentimiento y confidencialidad que existen al respecto. Dichas muestras podrán ser utilizadas en proyectos de investigación una vez validados convenientemente por los comités establecidos a tal fin.

\section{PAPEL DEL INTERNISTA EN OTRAS UNIDADES DE PACIENTES ADULTOS CON ENFERMEDADES RARAS}

El especialista en Medicina Interna, por su propia formación integral y por el desarrollo de su actividad asistencial en los hospitales, es una figura que debería te- ner un papel muy relevante en la atención a pacientes con ER, además de los ECM ya comentados. Además, existen muchas ER que prácticamente ya forman parte del ámbito de la medicina interna, por lo que la asunción cuando menos coordinadora del control de estos pacientes está probablemente fuera de discusión.

El Boletín Oficial del Estado de 7 de febrero de 2007 (Orden SCO/227/2007 de 24 de enero), en su apartado n.o 14 sobre los campos de acción del especialista en formación en Medicina Interna ya contempla, entre otros aspectos, la atención al paciente sin diagnóstico preciso o al enfermo con diversos procesos simultáneamente, la atención clínica a enfermos atendidos en unidades especiales de enfermedades metabólicas hereditarias, el cuidado de enfermos en fase paliativa de la enfermedad y la atención a pacientes con ER, ya sea por no inscribirse en una especialidad definida o por el desarrollo de nuevos conocimientos.

Por otro lado, las recomendaciones de la Unión Europea para afrontar el reto de las ER se enmarcan en los siguientes apartados:

1. Identificar expertos y crear redes (de diagnóstico, seguimiento y tratamientos) de los afectados y sus familiares.

2. Facilitar el acceso a los nuevos tratamientos huérfanos.

3. Simplificar los circuitos asistenciales y garantizar una buena conexión entre la atención primaria y los especialistas.

4. Potenciar prestaciones sociales (rehabilitación, ayudas).

5. Desplegar actividades formativas, informativas y de sensibilización dirigidas a profesionales sanitarios y sociales, a organizaciones de pacientes y al conjunto de la sociedad.

6. Potenciar la investigación biomédica y social en todos los ámbitos territoriales.

A modo de ejemplo, en la tabla 2 se expone un listado de ER en las que el internista puede tener un papel aglutinador y responsable de los pacientes que las sufren.

De la lectura de este texto se desprende, sin lugar a dudas, que el especialista en Medicina Interna puede intervenir muy activamente en distintos niveles de los especificados. De ningún modo se pretende insinuar que el internista deba ser el protagonista único e inequívoco de la solución del problema de las ER sino, como se ha dicho, que sea el facultativo de referencia coordinador que aglutine los esfuerzos de muchos profesionales de las diversas especialidades. 
Tabla 2. Relación de grandes grupos de ER en las que el internista puede desempeñar un papel relevante dentro del equipo asistencial

\begin{tabular}{|l|} 
Enfermedades Metabólicas Hereditarias (véase la tabla 1) \\
Porfirias \\
Enfermedades mitocondriales \\
Enfermedades Autoinmunes Sistémicas \\
Lupus eritematoso sistémico \\
Síndrome antifosfolipídico \\
Síndrome de Sjögren \\
Esclerosis sistémica (esclerodermia) \\
Sarcoidosis \\
Enfermedad de Behçet \\
Uveítis autoimmunes \\
Citopenias autoimmunes \\
Vasculitis \\
Enfermedades autoinflamatorias hereditarias \\
Enfermedades musculares \\
Distrofias musculares \\
Miopatías inflamatorias \\
Inmunodeficiencias
\end{tabular}

\section{AGRADECIMIENTOS}

FIPSE, CONACYT, Suport a grups de recerca y CERCA, programa de la Generalitat de Catalunya, Fundació la Marató de TV3, Fundació Cellex, CIBERER (iniciativa del ISCIII), Fondo de Investigación Sanitaria e InterCIBER.

\section{BIBLIOGRAFÍA}

Blum, R. W., Garell, D., Hodgman, C. H., Jorissen, T. W., Okinow, N. A., Orr, D. P. y Slap, G. B. (1993). Transition from ChildCentered to Adult Health-Care Systems for Adolescents with Chronic Conditions. A Position Paper of the Society for Adolescent Medicine. Journal of Adolescent Health, 14 (7), pp. 570-576. https://doi org/10.1016/1054-139X(93)90143-D

Buchbinder, N., Berger, M., Robert, A. y Vannier, J. P. (2011). Difficult transitions from paediatric to adult care in type 1 Gaucher disease. Archives de Pédiatrie. Organe officiel de la Societé Française de Pédiatrie, 18 (2), pp. 165-169. https:// doi.org/10.1016/j.arcped.2010.11.013

Cardellach, F. y Vilardell, M. (2006). Hacia el perfil de médico que necesita la comunidad. Medicina Clínica, 127 (4), pp. 136138. https://doi.org/10.1157/13090379

Grau, J. M. y Cardellach, F. (2010). Enfermedades raras y el especialista en Medicina Interna. Medicina Clínica, 134 (12), pp. 540-541. https://doi.org/10.1016/j. medcli.2010.01.021

Izquierdo Martínez, M. y Avellaneda Fernández, A. (2003). Enfoque multidisciplina- rio de las enfermedades raras: un nuevo reto para un nuevo siglo. Medicina Clínica, 121 (8), pp. 299-303. https://doi. org/10.1016/S0025-7753(03)73924-4

Kossoff, E. H., Veggiotti, P., Genton, P. Y Desguerre, I. (2014). Transition for patients with epilepsy due to metabolic and mitochondrial disorders. Epilepsia, 55 (supl. 3), pp. 37-40. https://doi. org/10.1111/epi.12709

Lee, P. J. (2002). Growing older: the adult metabolic clinic. Journal of Inherited Metabolic Disease, 25 (3), pp. 252-260. https://doi. org/10.1023/A:1015602601091

Mütze, U., Roth, A., Weigel, J. F. W., Beblo, S., Baerwald, C. G., Bührdel, P. y Kiess, W. (2011). Transition of young adults with phenylketonuria from pediatric to adult care. Journal of Inherited Metabolic Disease, 34 (3), pp. 701-709. https:// doi.org/10.1007/s10545-011-9284-x

Palau, F. (2010). Enfermedades raras, un paradigma emergente en la medicina del siglo XXI. Medicina Clínica, 134 (4), pp. 161-168. https://doi.org/10.1016/j. medcli.2009.06.038

\section{Disposiciones oficiales}

Orden SCO/227/2007 de 24 de enero, por la que se aprueba y publica el programa formativo de la especialidad de medicina interna. Boletín Oficial del Estado no 33 de 7 de febrero de 2007, pp. 57555759. Disponible en: https://www.boe. es/boe/dias/2007/02/07/pdfs/A0575505759.pdf

\section{Recursos en línea}

CatSalut. Servei Català de la Salut. Instrucció 12/2014, de 15 d'octubre de 2014 . Desarrollo e implantación del modelo de atención a las enfermedades minoritarias (EM) en Cataluña. Ordenación de las unidades expertas clínicas para EM o grupos temáticos de EM. [En línea]. Disponible en: http://catsalut.gencat. cat/web/.content/minisite/catsalut/ proveidors_professionals/normatives_instruccions/any_2014/instruccio_12_2014/instruccio_12_2014.pdf

Sociedad Española de Medicina Interna (SEMI). [En línea]. Disponible en: http:// www.fesemi.org/ 